\title{
Circulating microRNA-203 predicts metastases, early recurrence, and poor prognosis in human gastric cancer
}

\author{
Hiroki Imaoka $^{1} \cdot$ Yuji Toiyama $^{1} \cdot$ Masato Okigami $^{1} \cdot$ Hiromi Yasuda $^{1}$ • \\ Susumu Saigusa $^{1} \cdot$ Masaki Ohi $^{1} \cdot$ Koji Tanaka $^{1} \cdot$ Yasuhiro Inoue $^{1} \cdot$ \\ Yasuhiko Mohri ${ }^{1} \cdot$ Masato Kusunoki $^{1}$
}

Received: 1 December 2014 / Accepted: 19 July 2015/Published online: 2 August 2015

(c) The International Gastric Cancer Association and The Japanese Gastric Cancer Association 2015

\begin{abstract}
Background Metastasis is a major cause of death in patients with gastric cancer (GC). MicroRNAs (miRNAs) relating to the epithelial-mesenchymal transition (EMT) control GC progression and metastasis. The aim of this study was to evaluate serum EMT-associated miRNAs for metastatic and prognostic noninvasive biomarkers in GC.

Methods In the first step of this study (preliminary experiments), we selected candidate miRNAs associated with metastasis by analyzing the expression of the miR-200 family (miR-200a, miR-200b, miR-200c, miR-141, and miR-429) and miR-203 in serum samples from stage I $(n=12)$ and stage IV $(n=12)$ GC patients. The second phase involved the independent validation of candidate miRNAs in serum specimens from 130 patients with GC and 22 controls.

Results Based on the preliminary experiments, miR-203 was selected as the candidate serum miRNA that was most closely associated with metastasis. Validation analysis revealed that serum miR-203 levels were significantly lower in stage IV than stage I-III GC patients. Serum miR203 expression was significantly lower in GC patients with a higher $\mathrm{T}$ stage, vessel invasion, and lymph node, peritoneal, and distant metastases. Low expression of serum miR-203 was significantly associated with poor disease-
\end{abstract}

Electronic supplementary material The online version of this article (doi:10.1007/s10120-015-0521-0) contains supplementary material, which is available to authorized users.

Yuji Toiyama

ytoi0725@clin.medic.mie-u.ac.jp

1 Department of Gastrointestinal and Pediatric Surgery, Mie University Graduate School of Medicine, 2-174 Edobashi, Tsu, Mie 514-8507, Japan free and overall survival. Multivariate analysis revealed that low serum miR-203 expression was an independent predictive marker for lymph node, peritoneal, and distant metastases and a poor prognosis in patients with GC.

Conclusions Serum miR-203 has the potential to serve as a noninvasive biomarker for prognosis and to predict metastasis in patients with GC.

Keywords miR-203 - Gastric cancer · Serum ·

Noninvasive biomarker

\section{Introduction}

Gastric cancer (GC) is the second most common cause of cancer-related death worldwide, despite a decline in its incidence [1]. Diagnosis of gastric cancer (GC) is often delayed by a lack of early specific symptoms. Consequently, tumor invasion into the muscularis propria, socalled advanced GC, is found in many patients at the time of initial diagnosis. Metastasis of cancer cells is a critical event in tumor progression and for determining the prognosis of patients with GC. Recent advances in various therapeutic regimens that combine systemic chemotherapy with radiotherapy and surgery have contributed to improving the overall prognosis in advanced GC patients with various types of metastasis; nevertheless, their prognosis remains poor [2]. Therefore, the identification of predictive markers for cancer progression, metastasis, and prognosis would aid assessments of clinical outcome and potential treatment stratification for patients with GC.

MicroRNAs (miRNAs) are noncoding RNA molecules composed of 19-24 nucleotides that regulate gene expression by inhibiting or inactivating target messenger RNAs [3]. MiRNAs play a crucial role in diverse cellular 
biological processes, including differentiation, proliferation, growth, migration, and survival. The discovery that miRNA expression is frequently dysregulated in malignant tumors underpins their crucial role, which is a matter of active investigation, both from a basic science perspective and for its clinical usefulness [4]. Recently, several reports have highlighted the diagnostic and prognostic utility of serum or plasma-based miRNA levels, because tumorderived miRNAs are present in human circulation in remarkably stable forms that are protected from endogenous ribonuclease activity [5]. These studies suggest that serum or plasma miRNA-based assays may constitute accurate methods for the diagnosis and prognostic prediction of cancer, although, to date, only a few studies have specifically focused on the clinical significance of circulating miRNAs in patients with GC [6-12].

The epithelial-mesenchymal transition (EMT) manifests as the downregulation of E-cadherin and a loss of cell-to-cell adhesion, leading to a mesenchymal phenotype [13]. This contributes to accelerated invasiveness, dissemination, and metastasis of epithelial tumor cells in several carcinomas, including GC [14-16]. The miR-200 family (miR-200a, miR-200b, miR-200c, miR-141, and miR-429) and miR-203 inhibit E-cadherin suppressor targets such as ZEB1, ZEB2, and SNAI2, which are important initiators of EMT in various types of cancer $[17,18]$. Despite their involvement in metastasis, no study has investigated the clinical significance of the expression of the miR-200 family and miR-203 in sera of patients with GC.

Accordingly, in this study, we focused on analyzing the expression of the miR-200 family and miR-203 in the sera of patients with GC. First, in a small subset of samples, we selected candidate miRNAs that were associated with metastasis by comparing expression levels in the sera from patients with stage I and IV GC. Next, using a larger, independent cohort of serum specimens from patients with GC and healthy controls, we validated the clinical significance of selected miRNAs. Using this systematic approach, we aimed to demonstrate that serum levels of miR-203 are not only associated with a metastatic phenotype in GC but also serve as a potential biomarker for predicting lymph node metastasis, tumor recurrence, and prognosis in patients with GC.

\section{Materials and methods}

\section{Patients, study design, and clinical specimens}

Patients with GC who underwent surgical resection at the Mie University Hospital, Japan from January 2006 to December 2011 were enrolled in this study. In the preliminary experiments, we analyzed levels of the miR200 family and miR-203 in a subset of 24 serum samples from patients with stage I $(n=12)$ and stage IV $(n=12)$ GC. In the second step, candidate miRNAs that were significantly altered in the sera of patients with stage IV compared with stage I GC in the first step were validated in a larger, independent cohort, which included serum samples from $130 \mathrm{GC}$ patients and 22 normal controls. The patients included 91 men and 39 women with a median age of 68 years (standard deviation: 12.5 years). Thirteen patients were diagnosed with peritoneal metastasis and seven with synchronous liver metastasis. All patients were postoperatively classified based on histopathological analysis using the Union for International Cancer Control TNM staging system. There were 39 patients with stage I, 30 with stage II, 31 with stage III, and 30 with stage IV cancer. Seventy-nine patients had poorly differentiated or mucinous adenocarcinoma, and 51 had well or moderately differentiated adenocarcinoma. Postoperative follow-up data were obtained from all patients, and the median follow-up duration was 31.4 months (range 1-78 months). Basically, stage II-III GC patients who underwent R0 resection received S-1 (oral fluorinated pyrimidine) alone as postoperative adjuvant chemotherapy for 1 year, and the remaining stage IIIIV GC patients continued to receive S-1 and cisplatin therapy until disease progression was evident, unless patients were contraindicated by their conditions. When the tumor was refractory to S-1 and cisplatin, anticancer agents such as irinotecan or paclitaxel were administered until adverse effects occurred or further disease progression was confirmed. In addition, healthy volunteers were collected from a large pool of individuals seeking a routine health check-up at the Healthy Physical Examination Center of Mie University Hospital. People who showed no evidence of disease were selected as tumor-free healthy control subjects. Controls were matched to the patients based on age and sex. All participants in this study provided written informed consent according to the ethical guidelines approved by the institutional review board of Mie University Hospital, Japan, and indicated a willingness to donate their blood samples to our research.

\section{Total RNA isolation from serum and quantitative reverse transcription polymerase chain reaction (qRT-PCR)}

Small RNAs were enriched from all serum samples using the miRNeasy Mini Kit (Qiagen, Valencia, CA, USA). Briefly, $250 \mu \mathrm{L}$ of each serum sample were thawed on ice and centrifuged at $5000 \mathrm{rpm}$ for $5 \mathrm{~min}$ to remove cellular debris. Next, $100 \mu \mathrm{L}$ of supernatant were lysed with 5 volumes of QIAzol solution. To normalize for sample-to- 
sample variation during the RNA isolation procedures, 25 fmol of synthetic Caenorhabditis elegans miRNA (syn-celmiR-39) were added to each denatured sample. Small RNAs were enriched and purified according to the manufacturer's protocol, with the exception that the enriched small RNAs were eluted in $40 \mu \mathrm{L}$ RNase-free water. For miRNA-based RT-PCR assays, $2 \mu \mathrm{L}$ of enriched small RNAs from serum samples were reverse transcribed using the TaqMan MicroRNA Reverse Transcription Kit (Applied Biosystems, Foster City, CA, USA) in a total reaction volume of $15 \mu \mathrm{L}$, according to the manufacturer's instructions. RT products were diluted $1: 3$ and used as a PCR template. PCRs for quantitating miR-200a, miR-200b, miR-200c, miR-141, miR-429, miR-203, and cel-miR-39 were performed in duplicate using TaqMan Universal PCR Master Mix (Applied Biosystems), as described previously [19]. qRT-PCR was performed using the StepOnePlus Real Time PCR System (Applied Biosystems) with the following cycling conditions: $95{ }^{\circ} \mathrm{C}$ for $10 \mathrm{~min}, 45$ cycles at $95{ }^{\circ} \mathrm{C}$ for $15 \mathrm{~s}$, and $60{ }^{\circ} \mathrm{C}$ for $1 \mathrm{~min}$. Cycle threshold $(\mathrm{Ct})$ values were calculated using StepOne Software v2.2.2.

\section{Evaluation of miRNA expression}

The average expression levels of serum miRNAs were normalized with respect to syn-cel-miR-39 using the $2^{-\Delta C t}$ method. Differences between the groups are presented as $\Delta C t$, indicating the difference between the $C t$ value of the miRNA of interest and the $C t$ value of the normalizer miRNA. To ensure consistent measurements throughout all assays, for each PCR amplification reaction, three independent RNA samples were loaded as internal controls to account for any plate-to-plate variation, and the results from each plate were normalized against internal normalization controls.

\section{Statistical analysis}

All statistical analyses were performed using JMP version 10 (SAS Institute, Cary, NC, USA). The significance of each serum miRNA level was determined by the MannWhitney $U$ test, the Kruskal-Wallis test, or Pearson's $\chi^{2}$ test, where appropriate. Receiver operating characteristic (ROC) curves were established to determine cutoff values for analyzing predictions of metastasis, disease-free survival (DFS), and overall survival (OS) by Youden's index. The survival curves were obtained using the Kaplan-Meier method, and comparisons were made using the log-rank test. Prognostic factors were examined by univariate and multivariate analysis (Cox proportional hazards regression model). Logistic regression analysis was used to evaluate the factors influencing metastasis. All $P$ values were two- sided, and those $<0.05$ were considered statistically significant.

\section{Results}

\section{Serum miR-203 is a candidate miRNA that is associated with GC metastasis}

Among all of the miRNAs analyzed in the preliminary experiments, miR-203 was significantly suppressed in the sera of patients with stage IV compared with stage I GC (Fig. S1a in the Electronic supplementary material, ESM). In contrast, no significant differences were observed in serum miR-200a and miR-429 expression between patients with stage I and IV GC (Fig. S1b,c in the ESM). Expression levels ( $C t$ values) of the remaining miRNAs-miR200b, miR-200c, and miR-141 — could not be determined in this study. On the basis of these observations, we subsequently focused on validating and further exploring the clinical significance of miR-203 as a noninvasive biomarker in an independent set of serum samples from 130 GC patients and 22 healthy controls.

\section{Serum miR-203 expression level is associated with GC malignancy}

Expression levels of serum miR-203 in GC patients were significantly lower than in normal controls $(P<0.0001$; Fig. 1a). Furthermore, expression levels of miR-203 decreased in accordance with tumor TNM stage progression (Fig. 1b). Serum miR-203 expression in GC patients was divided into high or low groups using a cutoff value, which was determined as the maximum predictive value of serum miR-203 for OS based on ROC curve analysis (area under the curve $\mathrm{AUC}=0.707$, cutoff value $=0.0000067$ ) (Fig. S2a in the ESM). Fifty-three patients had high serum miR-203 expression and 77 had low expression. The relationship between miR-203 expression level in GC and clinicopathologic features is summarized in Table 1 . We divided the ages of the patients and tumor sizes by their median values (68 years and $45 \mathrm{~mm}$, respectively). Low miR-203 expression levels were significantly associated with male sex $(P=0.018)$, lymphatic invasion $(P=0.0004)$, venous invasion $(P=0.0057)$, high tumor depth $(P<0.0001)$, lymph node metastasis $(P<0.0001)$, liver metastasis $(P=0.0058)$, peritoneal metastasis $(P=0.0001)$, and distant metastasis $(P<0.0001)$. In addition, we showed the distribution of serum miR-203 subdivided by clinicopathological features, including histology, $\mathrm{T}$ stage, and presence or absence of hepatic, peritoneal, and distant metastasis (Fig. S2b-f in the ESM; 

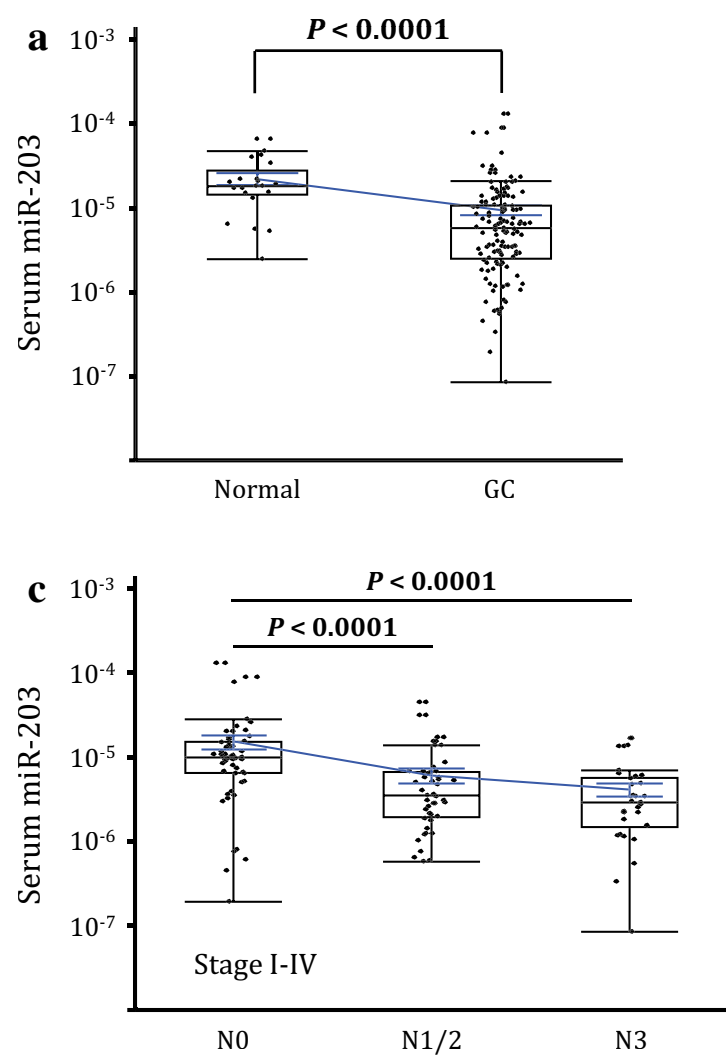

Fig. 1 Validation of miR-203 expression in a cohort of patients with stage I-IV GC. a Box plots of serum miR-203 expression levels in healthy normal controls $(n=22)$ and patients with GC $(n=130)$. b Box plots of serum miR-203 expression levels in patients with various stages of GC. Expression levels of serum miR-203 in patients with GC were significantly lower than in normal controls, and serum miR-203 levels in patients with stage IV GC were significantly lower than those in patients with stage I-III GC. c Box plots of serum miR-

Mann-Whitney $U$ test). These results are consistent with Table 1.

\section{Serum miR-203 level predicts GC patients with lymph node, peritoneal, and distant metastasis}

As described previously, low expression of serum miR-203 was associated with a metastatic phenotype, including lymph node, liver, and peritoneal metastasis, and the development of distant metastasis in patients with GC. We next analyzed serum miR-203 level in more detail on the basis of pathological extension of gastric neoplasia to lymph node metastasis. Serum miR-203 expression level was significantly lower in GC patients with lymph node metastasis of category $\mathrm{N} 1$ (one or two positive lymph nodes), N2 (3-6 positive lymph nodes: $P<0.0001$; Fig. 1c), or $\mathrm{N} 3$ ( $\geq 7$ positive lymph nodes: $P<0.0001$; Fig. 1c) than in those without lymph node metastasis (N0). Moreover, in stage I-III GC patients, serum miR-203 expression level decreased in accordance with the
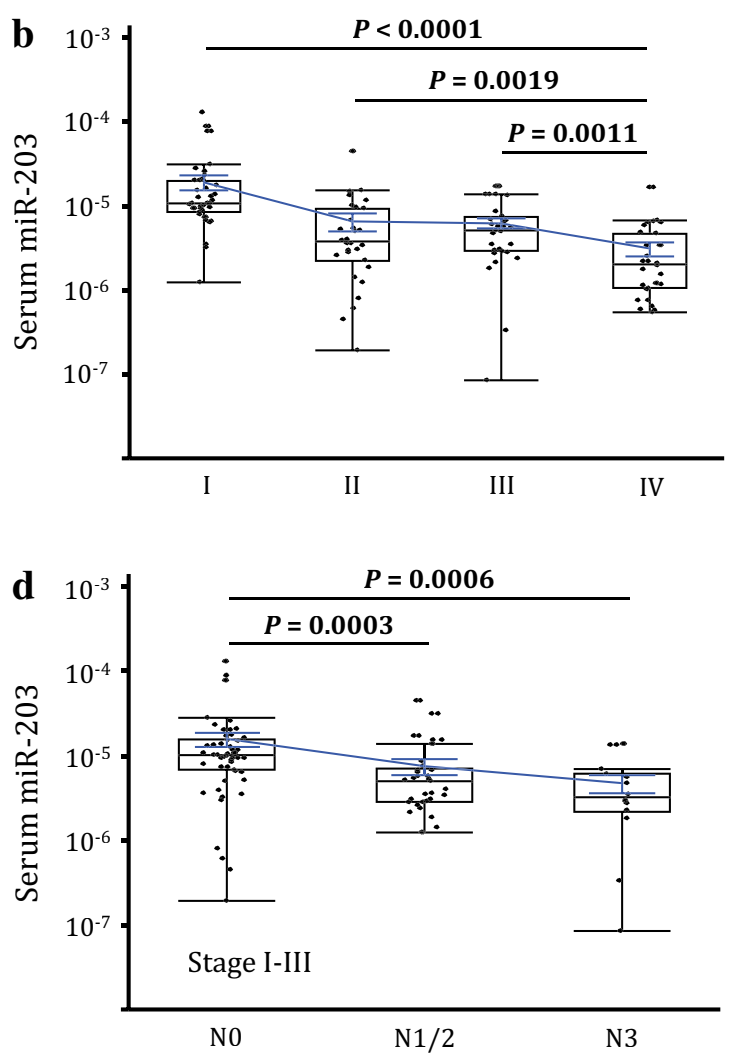

203 levels subdivided into patients with stage I-IV GC by N stage. d Box plots of serum miR-203 levels subdivided into patients with stage I-III GC by $\mathrm{N}$ stage. Expression levels of serum miR-203 decreased with increasing $\mathrm{N}$ stage. Expression levels of serum miR203 ( $\log 10$ scales on the $y$-axis) were normalized with respect to syncel-miR-39. Statistically significant differences were determined using the Mann-Whitney $U$ and Kruskal-Wallis tests

progression of lymph node metastasis, and miR-203 expression in $\mathrm{N} 1 / 2$ and $\mathrm{N} 3$ patients was significantly lower than that in N0 patients $(P=0.0003$ and $P=0.0006$, respectively; Fig. 1d). These results suggest that serum miR-203 levels in GC patients could be influenced by tumor volume or its dissemination to lymph node metastatic sites.

To evaluate further whether serum miR-203 levels can serve as a predictor of lymph node metastasis, we performed logistic regression analysis. Univariate analysis demonstrated that large tumor size $(\geq 45 \mathrm{~mm}$; $P=0.0004)$, with lymphatic $(P<0.0001)$ and venous $(P<0.0001)$ invasion, highly invasive tumors (T3/4; $P<0.0001)$, and low serum miR-203 expression $(P<0.0001)$ were significantly associated with lymph node metastasis in patients with stage I-IV GC (Table 2). Additionally, serum miR-203 expression was an independent predictive factor for lymph node metastasis in GC patients based upon multivariate logistic regression analysis [odds ratio (OR): 9.86, $95 \%$ confidence interval (CI): 
Table 1 Correlations between serum miR-203 levels and clinicopathological features in GC patients

\begin{tabular}{|c|c|c|c|c|}
\hline \multirow[t]{2}{*}{ Variables } & \multirow[t]{2}{*}{$n$} & \multicolumn{2}{|c|}{ Serum miR-203 expression $(n=130)$} & \multirow[t]{2}{*}{$P$ value } \\
\hline & & $\operatorname{High}(n=53)$ & Low $(n=77)$ & \\
\hline \multicolumn{5}{|l|}{ Age $(y)$} \\
\hline$<68$ & 64 & 31 & 33 & \multirow[t]{2}{*}{0.079} \\
\hline$\geq 68$ & 66 & 22 & 44 & \\
\hline \multicolumn{5}{|l|}{ Gender } \\
\hline Male & 91 & 31 & 60 & \multirow[t]{2}{*}{0.018} \\
\hline Female & 39 & 22 & 17 & \\
\hline \multicolumn{5}{|l|}{ Histology } \\
\hline Well/moderately differentiated & 51 & 21 & 30 & \multirow[t]{2}{*}{0.94} \\
\hline Poorly differentiated & 79 & 32 & 47 & \\
\hline \multicolumn{5}{|l|}{ Tumor size $(\mathrm{mm})$} \\
\hline$<45$ & 64 & 31 & 33 & \multirow[t]{2}{*}{0.079} \\
\hline$\geq 45$ & 66 & 22 & 44 & \\
\hline \multicolumn{5}{|l|}{ Lymphatic invasion } \\
\hline Negative & 23 & 17 & 6 & \multirow[t]{2}{*}{0.0004} \\
\hline Positive & 107 & 36 & 71 & \\
\hline \multicolumn{5}{|l|}{ Venous invasion } \\
\hline Negative & 67 & 35 & 32 & \multirow[t]{2}{*}{0.0057} \\
\hline Positive & 63 & 18 & 45 & \\
\hline \multicolumn{5}{|l|}{$\mathrm{T}$ classification } \\
\hline $\mathrm{T} 1 / \mathrm{T} 2$ & 52 & 37 & 15 & \multirow[t]{2}{*}{$<0.0001$} \\
\hline $\mathrm{T} 3 / \mathrm{T} 4$ & 78 & 16 & 62 & \\
\hline \multicolumn{5}{|l|}{ Lymph node metastasis } \\
\hline Negative & 55 & 40 & 15 & \multirow[t]{2}{*}{$<0.0001$} \\
\hline Positive & 75 & 13 & 62 & \\
\hline \multicolumn{5}{|l|}{ Liver metastasis } \\
\hline Negative & 123 & 53 & 70 & \multirow[t]{2}{*}{0.0058} \\
\hline Positive & 7 & 0 & 7 & \\
\hline \multicolumn{5}{|l|}{ Peritoneal metastasis } \\
\hline Negative & 117 & 53 & 64 & \multirow[t]{2}{*}{0.0001} \\
\hline Positive & 13 & 0 & 13 & \\
\hline \multicolumn{5}{|l|}{ Distant metastasis } \\
\hline Negative & 100 & 52 & 48 & \multirow[t]{2}{*}{$<0.0001$} \\
\hline Positive & 30 & 1 & 29 & \\
\hline \multicolumn{5}{|l|}{ TNM Stage } \\
\hline I & 39 & 34 & 5 & $<0.0001$ \\
\hline II & 30 & 9 & 21 & \\
\hline III & 31 & 9 & 22 & \\
\hline IV & 30 & 1 & 29 & \\
\hline
\end{tabular}

Median age and tumor size were 68 years and $45 \mathrm{~mm}$, respectively. Significant associations are shown in bold $(P<0.05)$

3.53-30.1; $P<0.0001$ ]. Likewise, we performed logistic analysis for lymph node metastasis in patients with stage IIII GC (Table 2) to investigate whether serum miR-203 can evaluate the status of lymph node metastasis preoperatively, enabling physicians to propose treatment strategies such as minimally invasive surgery or intensive neoadjuvant chemotherapy. Multivariate analysis showed that low expression level of serum miR-203 was an independent predictor of lymph node metastasis in this patient group (OR: 8.06, $95 \%$ CI: 2.73-26.59; $P=0.0001$ : Table 2). Similarly, we evaluated the potential of serum miR-203 for predicting other metastatic phenotypes, including peritoneal dissemination and distant metastasis. Univariate analysis demonstrated that large tumor size $(\geq 45 \mathrm{~mm}$; 
Table 2 Univariate and multivariate analyses of predictive factors for lymph node metastasis in patients with GC (logistic regression model)

\begin{tabular}{|c|c|c|c|c|c|c|}
\hline \multirow[b]{2}{*}{ Stage I-IV (variables) } & \multicolumn{3}{|c|}{ Univariate analysis } & \multicolumn{3}{|c|}{ Multivariate analysis } \\
\hline & Odds ratio & $95 \% \mathrm{CI}$ & $P$ value & Odds ratio & $95 \% \mathrm{CI}$ & $P$ value \\
\hline Histology (poorly vs well/moderately differentiated) & 1.21 & $0.59-2.46$ & 0.61 & - & - & - \\
\hline Tumor size $(\geq 45 \mathrm{~mm}$ vs $<45 \mathrm{~mm})$ & 3.65 & $1.78-7.75$ & 0.0004 & 2.76 & $0.97-8.19$ & 0.058 \\
\hline Lymphatic invasion (positive vs negative) & 49.33 & $9.72-901.55$ & $<0.0001$ & 22.83 & $3.57-458.60$ & 0.0003 \\
\hline Venous invasion (positive vs negative) & 4.74 & $2.26-10.36$ & $<0.0001$ & 1.61 & $0.57-4.54$ & 0.36 \\
\hline $\mathrm{T}$ classification (T3/T4 vs $\mathrm{T} 1 / \mathrm{T} 2)$ & 8.22 & $3.78-18.79$ & $<0.0001$ & 1.38 & $0.43-4.26$ & 0.58 \\
\hline Serum miR-203 expression (low vs high) & 12.72 & $5.64-30.63$ & $<0.0001$ & 9.86 & $3.53-30.10$ & $<0.0001$ \\
\hline \multicolumn{7}{|l|}{ Stage I-III (variables) } \\
\hline Histology (poorly vs well/moderately differentiated) & 1.51 & $0.67-3.49$ & 0.32 & - & - & - \\
\hline Tumor size $(\geq 35 \mathrm{~mm}$ vs $<35 \mathrm{~mm})$ & 4.22 & $1.85-10.02$ & 0.0005 & 2.80 & $0.90-9.10$ & 0.076 \\
\hline Lymphatic invasion (positive vs negative) & 32.65 & $6.34-599.43$ & $<0.0001$ & 18.90 & $2.96-378.80$ & 0.0008 \\
\hline Venous invasion (positive vs negative) & 3.49 & $1.52-8.36$ & 0.003 & 1.42 & $0.46-4.49$ & 0.54 \\
\hline $\mathrm{T}$ classification (T3/T4 vs $\mathrm{T} 1 / \mathrm{T} 2)$ & 5.45 & $2.36-13.21$ & $<0.0001$ & 1.27 & $0.38-4.06$ & 0.69 \\
\hline Serum miR-203 expression (low vs high) & 8.97 & $3.74-23.09$ & $<0.0001$ & 8.06 & $2.73-26.59$ & 0.0001 \\
\hline
\end{tabular}

Median tumor size was $45 \mathrm{~mm}$ in stage I-IV and $35 \mathrm{~mm}$ in stage I-III. Significant associations are shown in bold $(P<0.05)$

CI confidence interval

$P=0.0006), \quad$ lymphatic $\quad(P=0.021) \quad$ and $\quad$ venous $(P=0.0042)$ invasion, highly invasive tumors (T3/4; $P=0.0002$ ), positive lymph node metastasis $(P=0.0034)$, and low serum miR-203 expression $(P=0.0001)$ were significantly associated with peritoneal dissemination in GC patients (Table 3). Additionally, serum miR-203 expression was an independent predictive factor for peritoneal dissemination in GC patients based upon multivariate logistic regression analysis $(P=0.017$ : Table 3). Regarding distant metastasis, large tumor size $(\geq 45 \mathrm{~mm} ; \quad P=0.045)$, lymphatic $(P=0.0002)$ and venous $(P<0.0001)$ invasion, highly invasive tumors (T3/ 4; $\quad P<0.0001)$, positive lymph node metastasis $(P<0.0001)$, and low levels of serum miR-203 expression $(P<0.0001)$ were significantly associated with distant metastasis (Table 4). Consequently, low expression of serum miR-203 was selected as an independent factor for distant metastasis in GC patients (OR: 12.04, $95 \% \mathrm{CI}$ : 2.07-230.05; $P=0.0032$; Table 4).

\section{Serum miR-203 expression level predicts GC patient prognosis}

Kaplan-Meier analysis showed that GC patients with low miR-203 expression had significantly poorer OS and DFS than those with high miR-203 expression (Fig. 2a, b) ( $P<0.0001$ and $P=0.021$, respectively; log-rank test). To determine whether serum miR-203 expression was an independent risk factor for prognosis, the Cox proportional hazard regression model was used (Table 5). Univariate analysis showed that age $(\geq 68$ years; $P=0.017)$, lymphatic invasion $(P=0.032)$, venous invasion

Table 3 Univariate and multivariate analyses of predictive factors for peritoneal metastasis in patients with GC (logistic regression model)

\begin{tabular}{|c|c|c|c|c|c|c|}
\hline \multirow[t]{2}{*}{ Variables } & \multicolumn{3}{|c|}{ Univariate analysis } & \multicolumn{3}{|c|}{ Multivariate analysis } \\
\hline & Odds ratio & $95 \% \mathrm{CI}$ & $P$ value & Odds ratio & $95 \% \mathrm{CI}$ & $P$ value \\
\hline Histology (poorly vs well/moderately differentiated) & 2.31 & $0.67-10.74$ & 0.19 & - & - & - \\
\hline Tumor size $(\geq 45 \mathrm{~mm}$ vs $<45 \mathrm{~mm})$ & 14.0 & $2.63-259.09$ & 0.0006 & 6.98 & $1.17-134.02$ & 0.031 \\
\hline Lymphatic invasion (positive vs negative) & NA & NA & 0.021 & NA & NA & 0.78 \\
\hline Venous invasion (positive vs negative) & 6.88 & $1.75-45.70$ & 0.0042 & 2.04 & $0.43-14.86$ & 0.37 \\
\hline $\mathrm{T}$ classification (T3/T4 vs $\mathrm{T} 1 / \mathrm{T} 2)$ & NA & NA & 0.0002 & NA & NA & 0.19 \\
\hline Lymph node metastasis (positive vs negative) & 10.29 & $1.93-190.35$ & 0.0034 & 1.69 & $0.22-34.92$ & 0.64 \\
\hline Serum miR-203 expression (low vs high) & NA & NA & 0.0001 & NA & NA & 0.017 \\
\hline
\end{tabular}

Median tumor size was $45 \mathrm{~mm}$. Significant associations are shown in bold $(P<0.05)$

CI confidence interval; $N A$ not available 
Table 4 Univariate and multivariate analyses of predictive factors for distant metastasis in patients with GC (logistic regression model)

\begin{tabular}{|c|c|c|c|c|c|c|}
\hline \multirow[t]{2}{*}{ Variables } & \multicolumn{3}{|c|}{ Univariate analysis } & \multicolumn{3}{|c|}{ Multivariate analysis } \\
\hline & Odds ratio & $95 \% \mathrm{CI}$ & $P$ value & Odds ratio & $95 \% \mathrm{CI}$ & $P$ value \\
\hline Histology (poorly vs well/moderately differentiated) & 1.49 & $0.65-3.41$ & 0.34 & - & - & - \\
\hline Tumor size $(\geq 45 \mathrm{~mm}$ vs $<45 \mathrm{~mm})$ & 2.35 & $1.02-5.71$ & 0.045 & 1.32 & $0.43-4.23$ & 0.63 \\
\hline Lymphatic invasion (positive vs negative) & NA & NA & 0.0002 & NA & NA & 0.43 \\
\hline Venous invasion (positive vs negative) & 8.16 & $3.09-25.80$ & $<0.0001$ & 3.77 & $1.18-13.80$ & 0.025 \\
\hline $\mathrm{T}$ classification $(\mathrm{T} 3 / \mathrm{T} 4$ vs $\mathrm{T} 1 / \mathrm{T} 2)$ & 30.18 & $6.08-547.79$ & $<0.0001$ & 7.03 & $1.03-142.28$ & 0.046 \\
\hline Lymph node metastasis (positive vs negative) & 15.79 & $4.42-101.05$ & $<0.0001$ & 4.39 & $0.94-32.36$ & 0.061 \\
\hline Serum miR-203 expression (low vs high) & 31.42 & $6.33-570.18$ & $<0.0001$ & 12.04 & $2.07-230.05$ & 0.0032 \\
\hline
\end{tabular}

Median tumor size was $45 \mathrm{~mm}$. Significant associations are shown in bold $(P<0.05)$

CI confidence interval; NA not available

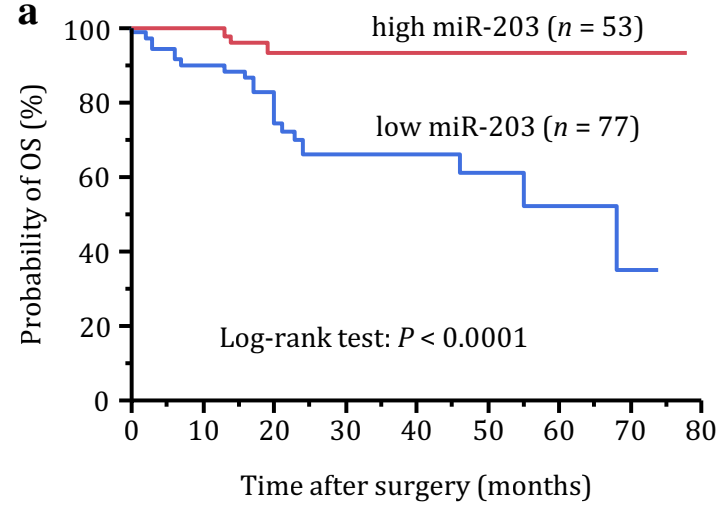

Fig. 2 Kaplan-Meier survival curves of patients with GC according to the status of serum miR-203 expression levels. a Patients with low expression levels of serum miR-203 showed significantly poorer OS than those with high expression levels of serum miR-203 $(P<0.0001, \log$-rank test). b Patients with low expression levels

( $P=0.0011)$, high T stage (T3/4; $P=0.004)$, lymph node metastasis $(P<0.0001)$, high TNM stage (stage III/IV; $P<0.0001)$, and low serum miR-203 expression $(P<0.0001)$ were significantly associated with poor OS. Furthermore, multivariate analysis showed that venous invasion [hazard ratio (HR): 3.92, $95 \%$ CI: 1.19-18.53, $P=0.022$ ], high $\mathrm{T}$ stage (HR: 18.42, $95 \% \quad \mathrm{CI}$ : 2.12-157.65, $P=0.0082$ ), high TNM stage (HR: 23.09, $95 \%$ CI: $2.81-233.90, P=0.0024)$, and low serum miR203 expression (HR: 4.51, $95 \%$ CI: 1.23-23.69, $P=0.021$ ) were independent prognostic markers for predicting poor prognosis in patients with GC.

\section{Discussion}

MiRNAs serve as major regulators of gene expression through their ability to bind and post-transcriptionally mediate the expression of targeted mRNAs [20]. miRNAs play a central role in cellular differentiation, development,

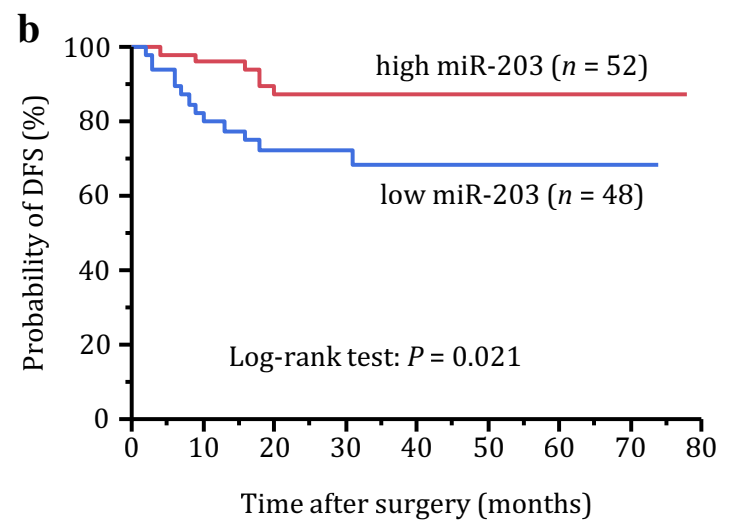

of serum miR-203 showed poorer DFS than those with low expression levels of serum miR-203 ( $P=0.021, \log$-rank test). Cutoff thresholds for miR-203 expression in serum were deduced from ROC curves with Youden's index

proliferation, and apoptosis in a variety of cell types, including cancer cells. In cancer, all of these processes are deregulated through altered expression of miRNAs, suggesting that miRNAs are involved in carcinogenesis and contribute to the initiation and progression of cancer [21].

Although many studies have reported the aberrant expression of miRNAs in GC, almost all these studies have focused on the expression of miRNA in tumor tissues and cell lines. Although miRNAs expressed in the tissue may provide an accurate diagnosis for various types of cancer, the difficulty or invasiveness involved in collecting tissue samples limits their application for the detection of cancer biomarkers. Sampling tissue specimens requires an invasive procedure and surgical resection after initial clinical classification. Studies aimed at establishing noninvasive diagnostic tools for neoplasia have long been a goal of many researchers, so much of the interest in this field has been in the circulation of nucleic acids in serum or plasma. Endogenous miRNAs in serum or plasma have been shown to remain stable, even when subjected to extreme 
Table 5 Univariate and multivariate analyses of predictive factors for prognosis in patients with GC (Cox proportional hazards regression model)

\begin{tabular}{|c|c|c|c|c|c|c|}
\hline \multirow[t]{2}{*}{ Variables } & \multicolumn{3}{|c|}{ Univariate analysis } & \multicolumn{3}{|c|}{ Multivariate analysis } \\
\hline & HR & $95 \% \mathrm{CI}$ & $P$ value & HR & $95 \% \mathrm{CI}$ & $P$ value \\
\hline Age ( $\geq 68$ vs $<68$ years $)$ & 2.68 & $1.19-6.58$ & $\mathbf{0 . 0 1 7}$ & 1.73 & $0.72-4.66$ & 0.23 \\
\hline Gender (male vs female) & 1.43 & $0.60-3.94$ & 0.43 & - & - & - \\
\hline Histology (poorly vs well/moderately differentiated) & 1.10 & $0.50-2.61$ & 0.81 & - & - & - \\
\hline Lymphatic invasion (positive vs negative) & 3.74 & $1.10-23.37$ & $\mathbf{0 . 0 3 2}$ & 0.29 & $0.032-2.74$ & 0.26 \\
\hline Venous invasion (positive vs negative) & 4.06 & $1.71-11.15$ & 0.0011 & 3.92 & $1.19-18.53$ & 0.022 \\
\hline $\mathrm{T}$ classification (T3/T4 vs $\mathrm{T} 1 / \mathrm{T} 2)$ & 3.62 & $1.48-10.30$ & 0.004 & 18.42 & $2.12-157.65$ & 0.0082 \\
\hline Lymph node metastasis (positive vs negative) & 10.44 & $3.52-44.83$ & $<0.0001$ & 3.64 & $0.77-24.10$ & 0.11 \\
\hline TNM stage (III/IV vs I/II) & 10.16 & $3.73-35.82$ & $<0.0001$ & 23.09 & $2.81-233.90$ & 0.0024 \\
\hline Serum miR-203 expression (low vs high) & 7.92 & $2.71-33.70$ & $<0.0001$ & 4.51 & $1.23-23.69$ & 0.021 \\
\hline
\end{tabular}

Median age and tumor size were 68 years and $45 \mathrm{~mm}$, respectively. Significant associations are shown in bold $(P<0.05)$

$H R$ hazard ratio, $C I$ confidence interval

conditions such as boiling, low or high $\mathrm{pH}$ levels, extended storage time, and multiple freeze-thaw cycles [5, 22]. These unique features of miRNAs in body fluids represent attractive advantages of these new and more useful noninvasive biomarkers for cancer [23].

In this study, we investigated the potential clinical utility of miR-200 family members and miR-203, which are thought to be involved in EMT [17, 18, 24-27]; i.e., whether these miRNAs can serve as noninvasive prognostic and metastasis-predictive biomarkers in patients with GC. To this end, in a small subset of serum specimens, we found that serum miR-203 levels in stage IV GC were significantly lower than those in stage I patients. Therefore, we selected miR-203 and subsequently validated this miRNA as a candidate serum miRNA with potential as a predictive biomarker for metastasis in GC. We performed independent validation experiments using a large cohort of samples from $130 \mathrm{GC}$ patients and 22 control subjects. These data provided strong evidence that serum miR-203 levels in patients with GC were significantly lower than those in healthy controls. Moreover, serum miR-203 levels were significantly lower in patients with stage IV GC than in those with stage I-III GC. In addition, low expression levels of serum miR-203 were associated with poorer OS and DFS, and served as an independent prognostic biomarker in patients with GC.

Our data are of particular interest because they highlight that low expression levels of serum miR-203 provide independent predictors of lymph node, peritoneal, and distant metastasis, respectively. In particular, it is still too difficult to diagnose peritoneal dissemination, regardless of recent diagnostic improvements. Thus, accurate preoperative diagnosis of peritoneal metastasis permits the selection of GC patients who may benefit from neoadjuvant chemotherapy or hyperthermic intraperitoneal chemotherapy followed by cytoreductive surgery. More importantly, the decrease in serum miR-203 levels can discriminate patients with from those without lymph node metastasis. Currently, there are several therapeutic options, including endoscopic mucosal resection, endoscopic submucosal dissection, and surgical gastrectomy with regional lymphadenectomy. For the selection of endoscopic treatment, suitable patients should be identified who are predicted to have no lymph node metastasis after investigating macroscopic morphological type, tumor size, presence of ulcer, and histological type in biopsy specimens. Considering the clinical challenge that a significant proportion of these patients are erroneously misdiagnosed using these conventional pathological criteria, incorporating more robust molecular biomarkers, such as the quantification of miR-203 expression in serum, followed by the preoperative selection of patients without lymph node metastasis, could promote minimally invasive treatment of early GC.

MiR-203 is reported to function as a tumor-suppressive miRNA in many types of cancer studied in tissues and cell lines [28-33]. Ectopic expression of miR-203 in prostate cancer cell lines could influence proliferation, apoptosis, and migration [32], and overexpression of miR-203 in laryngeal carcinoma cells reduces cell viability and leads to cell-cycle arrest in G1 phase [31]. Additionally, expression of miR-203 suppresses cell proliferation and migration in human triple-negative breast cancer cells [29], and miR203 suppresses proliferation and migration and promotes apoptosis of lung cancer cells by targeting the SRC gene [28]. In cancer metastasis, EMT is a key process that converts polarized immotile epithelial cells into motile invasive mesenchymal cells, which subsequently enables cancer cells to acquire stem cell characteristics and aggressive malignant properties [34, 35]. MiR-203 has been shown to directly suppress EMT activators such as 
ZEB2 and SNAI1/2 in various types of cancer [32, 36-38]. Regarding miR-203 expression in GC tissue, Chiang et al. revealed that, although expression of miR-203 did not differ significantly between GC tissues and normal gastric mucosa, low expression of miR-203 was significantly correlated with large tumor size, macroscopic type, and advanced pathological T stage [39]. Moreover, Saad et al. reported a tendency for lower miR-203 expression in GC tissue than normal gastric tissue, even though that study focused on a small patient cohort [40]. In this way, it is speculated that the downregulation of miR-203 expression in GC accelerates EMT phenomena and tumor aggressiveness as well as other types of cancer. However, the clinical importance of serum miR-203 has not been investigated at all. Therefore, our investigation of serum miR-203 expression affords new insight into noninvasive prognostic and metastatic biomarkers in GC patients.

In conclusion, we have provided novel evidence of serum miR-203 expression as a robust biomarker for prognosis and metastasis of GC. GC patients with low expression of serum miR-203 had worse OS and DFS, which highlights that identifying serum miR-203 expression would be potentially helpful for identifying patients who have a high risk of recurrence and need adjuvant chemotherapy and strict surveillance. In addition, serum miR-203 expression predicts several metastatic phenotypes preoperatively, including peritoneal and distant metastasis, which can aid the selection of patients who are suitable for multidisciplinary therapy. Furthermore, we verified the clinical significance of serum miR-203 as a novel predictive biomarker of lymph node metastasis, meaning that it could help reduce GC-related mortality and morbidity by indicating patients who would benefit from minimally invasive and curative treatment during the earlier stages of GC. However, the reproducibility of our results need to be confirmed by performing an appropriate retrospective study on a large cohort. In addition, in order to confirm the suitability of this biomarker for clinical application, a prospective study focusing on whether serum miR-203 can indeed identify GC patients with poor prognosis, early recurrence, and several metastases should be carried out.

Acknowledgments This work was supported in part by Grants-inAid for Scientific Research (nos. 23591926, 25462018, 26461976) from the Ministry of Education, Culture, Sports, Science, and Technology, Japan. This study was also supported by Grant Aid for Cancer Research from the Mie Health Center, Japan.

\section{References}

1. Siegel R, Naishadham D, Jemal A. Cancer statistics, 2012. CA Cancer J Clin. 2012;62:10-29.
2. Takahashi T, Saikawa Y, Kitagawa Y. Gastric cancer: current status of diagnosis and treatment. Cancers (Basel). 2013;5:48-63.

3. Cortez MA, Calin GA. MicroRNA identification in plasma and serum: a new tool to diagnose and monitor diseases. Expert Opin Biol Ther. 2009;9:703-11.

4. van Kouwenhove M, Kedde M, Agami R. MicroRNA regulation by RNA-binding proteins and its implications for cancer. Nat Rev Cancer. 2011;11:644-56.

5. Mitchell PS, Parkin RK, Kroh EM, Fritz BR, Wyman SK, Pogosova-Agadjanyan EL, et al. Circulating microRNAs as stable blood-based markers for cancer detection. Proc Natl Acad Sci USA. 2008;105:10513-8.

6. Gorur A, Balci Fidanci S, Dogruer Unal N, Ayaz L, Akbayir S, Yildirim Yaroglu $\mathrm{H}$, et al. Determination of plasma microRNA for early detection of gastric cancer. Mol Biol Rep. 2013;40:2091-6.

7. Wang M, Gu H, Wang S, Qian H, Zhu W, Zhang L, et al. Circulating miR-17-5p and miR-20a: molecular markers for gastric cancer. Mol Med Rep. 2012;5:1514-20.

8. Tsai KW, Liao YL, Wu CW, Hu LY, Li SC, Chan WC, et al. Aberrant expression of miR-196a in gastric cancers and correlation with recurrence. Genes Chromosom Cancer. 2012;51:394-401.

9. Song MY, Pan KF, Su HJ, Zhang L, Ma JL, Li JY, et al. Identification of serum microRNAs as novel non invasive biomarkers for early detection of gastric cancer. PLoS One. 2012;7:e33608.

10. Liu H, Zhu L, Liu B, Yang L, Meng X, Zhang W, et al. Genomewide microRNA profiles identify miR-378 as a serum biomarker for early detection of gastric cancer. Cancer Lett. 2012;316:196-203.

11. Liu R, Zhang C, Hu Z, Li G, Wang C, Yang C, et al. A fivemicroRNA signature identified from genome-wide serum microRNA expression profiling serves as a fingerprint for gastric cancer diagnosis. Eur J Cancer. 2011;47:784-91.

12. Kim SY, Jeon TY, Choi CI, Kim DH, Kim DH, Kim GH, et al. Validation of circulating miRNA biomarkers for predicting lymph node metastasis in gastric cancer. J Mol Diagn. 2013;15:661-9.

13. Thiery JP, Acloque H, Huang RY, Nieto MA. Epithelial-mesenchymal transitions in development and disease. Cell. 2009;139:871-90.

14. Okugawa Y, Toiyama Y, Tanaka K, Matsusita K, Fujikawa H, Saigusa $S$, et al. Clinical significance of zinc finger e-box binding homeobox 1 (ZEB1) in human gastric cancer. J Surg Oncol. 2012;106:280-5.

15. Castro Alves C, Rosivatz E, Schott C, Hollweck R, Becker I, Sarbia M, et al. Slug is overexpressed in gastric carcinomas and may act synergistically with SIP1 and Snail in the down-regulation of E-cadherin. J Pathol. 2007;211:507-15.

16. Rosivatz E, Becker I, Specht K, Fricke E, Luber B, Busch R, et al. Differential expression of the epithelial-mesenchymal transition regulators snail, SIP1, and twist in gastric cancer. Am J Pathol. 2002;161:1881-91.

17. Burk U, Schubert J, Wellner U, Schmalhofer O, Vincan E, Spaderna S, et al. A reciprocal repression between ZEB1 and members of the miR-200 family promotes EMT and invasion in cancer cells. EMBO Rep. 2008;9:582-9.

18. Ding X, Park SI, McCauley LK, Wang CY. Signaling between transforming growth factor beta (TGF-beta) and transcription factor SNAI2 represses expression of microRNA miR-203 to promote epithelial-mesenchymal transition and tumor metastasis. J Biol Chem. 2013;288:10241-53.

19. Kroh EM, Parkin RK, Mitchell PS, Tewari M. Analysis of circulating microRNA biomarkers in plasma and serum using quantitative reverse transcription-PCR (qRT-PCR). Methods. 2010;50:298-301. 
20. Lagos-Quintana M, Rauhut R, Lendeckel W, Tuschl T. Identification of novel genes coding for small expressed RNAs. Science. 2001;294:853-8.

21. Taft RJ, Pang KC, Mercer TR, Dinger M, Mattick JS. Non coding RNAs: regulators of disease. J Pathol. 2010;220:126-39.

22. Chen X, Ba Y, Ma L, Cai X, Yin Y, Wang K, et al. Characterization of microRNAs in serum: a novel class of biomarkers for diagnosis of cancer and other diseases. Cell Res. 2008;18:997-1006.

23. Fabbri M. miRNAs as molecular biomarkers of cancer. Expert Rev Mol Diagn. 2010;10:435-44.

24. Park SM, Gaur AB, Lengyel E, Peter ME. The miR-200 family determines the epithelial phenotype of cancer cells by targeting the E-cadherin repressors ZEB1 and ZEB2. Genes Dev. 2008;22:894-907.

25. Korpal M, Lee ES, Hu G, Kang Y. The miR-200 family inhibits epithelial-mesenchymal transition and cancer cell migration by direct targeting of E-cadherin transcriptional repressors ZEB1 and ZEB2. J Biol Chem. 2008;283:14910-4.

26. Gregory PA, Bert AG, Paterson EL, Barry SC, Tsykin A, Farshid $\mathrm{G}$, et al. The miR-200 family and miR-205 regulate epithelial to mesenchymal transition by targeting ZEB1 and SIP1. Nat Cell Biol. 2008;10:593-601.

27. Wellner U, Schubert J, Burk UC, Schmalhofer O, Zhu F, Sonntag A, et al. The EMT-activator ZEB1 promotes tumorigenicity by repressing stemness-inhibiting microRNAs. Nat Cell Biol. 2009;11:1487-95.

28. Wang N, Liang H, Zhou Y, Wang C, Zhang S, Pan Y, et al. miR203 suppresses the proliferation and migration and promotes the apoptosis of lung cancer cells by targeting SRC. PLoS One. 2014;9:e105570.

29. Wang C, Zheng X, Shen C, Shi Y. MicroRNA-203 suppresses cell proliferation and migration by targeting BIRC5 and LASP1 in human triple-negative breast cancer cells. J Exp Clin Cancer Res. 2012;31:58.

30. Takeshita N, Mori M, Kano M, Hoshino I, Akutsu Y, Hanari N, et al. miR-203 inhibits the migration and invasion of esophageal squamous cell carcinoma by regulating LASP1. Int $\mathrm{J}$ Oncol. 2012;41:1653-61.

31. Bian K, Fan J, Zhang X, Yang XW, Zhu HY, Wang L, et al. MicroRNA-203 leads to G1 phase cell cycle arrest in laryngeal carcinoma cells by directly targeting survivin. FEBS Lett. 2012;586:804-9.

32. Saini S, Majid S, Yamamura S, Tabatabai L, Suh SO, Shahryari $\mathrm{V}$, et al. Regulatory role of mir-203 in prostate cancer progression and metastasis. Clin Cancer Res. 2011;17:5287-98.

33. Abella V, Valladares M, Rodriguez T, Haz M, Blanco M, Tarrio $\mathrm{N}$, et al. miR-203 regulates cell proliferation through its influence on Hakai expression. PLoS One. 2012;7:e52568.

34. Jou J, Diehl AM. Epithelial-mesenchymal transitions and hepatocarcinogenesis. J Clin Invest. 2010;120:1031-4.

35. Yang J, Weinberg RA. Epithelial-mesenchymal transition: at the crossroads of development and tumor metastasis. Dev Cell. 2008; $14: 818-29$.

36. Moes M, Le Bechec A, Crespo I, Laurini C, Halavatyi A, Vetter $\mathrm{G}$, et al. A novel network integrating a miRNA-203/SNAI1 feedback loop which regulates epithelial to mesenchymal transition. PLoS One. 2012;7:e35440.

37. Qu Y, Li WC, Hellem MR, Rostad K, Popa M, McCormack E, et al. MiR-182 and miR-203 induce mesenchymal to epithelial transition and self-sufficiency of growth signals via repressing SNAI2 in prostate cells. Int J Cancer. 2013;133:544-55.

38. Zhang Z, Zhang B, Li W, Fu L, Fu L, Zhu Z, et al. Epigenetic silencing of miR-203 upregulates SNAI2 and contributes to the invasiveness of malignant breast cancer cells. Genes Cancer. 2011;2:782-91.

39. Chiang Y, Song Y, Wang Z, Chen Y, Yue Z, Xu H, et al. Aberrant expression of miR-203 and its clinical significance in gastric and colorectal cancers. J Gastrointest Surg. 2011;15:63-70.

40. Saad R, Chen Z, Zhu S, Jia P, Zhao Z, Washington MK, et al. Deciphering the unique microRNA signature in human esophageal adenocarcinoma. PLoS One. 2013;8:e64463. 